УДК 537.611.2, 537.874.7

\title{
Influence of Dissipation on Magnetostatic Surface Waves Dispersion
}

\author{
Pavel A. Makarov* \\ Institute of Exact Sciences and Information Technology \\ Pitirim Sorokin' Syktyvkar State University \\ Oktyabrsky, 55, Syktyvkar, 167001 \\ Russia \\ Vladimir I. Shcheglov ${ }^{\dagger}$ \\ Institute of Radio-Engineering and Electronics of RAS \\ Mokhovaya, 11-7, Moscow, 125009 \\ Russia
}

Received 21.08.2016, received in revised form 08.10.2016, accepted 14.11.2016

By means of consistent solution of the motion equation for magnetization the consideration of energy dissipation for magnetostatic surface waves propagating on in-plane magnetized ferrite plate is regarded. In this case the dispersion relations for the real and imaginary parts of the wave number are obtained. It is presented that dispersion curves are limited both by the wave number and frequency, and these restrictions tighten with an increase in the damping parameter. Both for forward and backward waves there is a critical value of the damping parameter and propagation angle, beyond which it does not exist.

Keywords: dispersion, ferrites, magnetostatic waves, dissipation.

DOI: 10.17516/1997-1397-2016-9-4-469-472.

\section{Introduction}

Magnetostatic surface waves (MSSW) propagating in ferrite films are the basis for the construction of number microwave devices for analog data processing, information transfer and control of high frequency dynamics [1-4]. Minimization of wave losses is a key requirement for its correct work, however the MSSW damping is not studied enough as the vast majority of research is performed without consider attenuation [4-6]. Although, the damping may significantly transform the dispersion law [7]. In particular, it limits the maximum value of the wave number, which results in reasing the minimum value of the signal delay time.

In the most invesigations [3-7], propagation of MSSW is studied in the special case when wave is transferred perpendicularly to the direction of the permanent external magnetic field. However, the wave propagation at arbitrary angles is essential for some devices, such as multichannel filters $[1,2]$. Hence, the aim of this research is to analize the influence the damping effect on the dispersion of MSSW at arbitrary angles.

\footnotetext{
*makarovpa@syktsu.ru

†vshcheg@cplire.ru

(c) Siberian Federal University. All rights reserved
} 


\section{Brief theory}

The geometry of the problem is the following. An unbounded ferrite slab of thickness $d$ is magnetized to saturation $4 \pi \vec{M}$ by the constant field $\vec{H}$. The Cartesian coordinates Oxyz are chosen so as the origin is at the middle of the slab thickness, the $O y z$ plane is parallel to the plane of the slab, and the axis $O x$ is perpendicular to latter. The $O z$ axis is oriented along the field $\vec{H}$. The wave vector $\vec{k}$ lies on the $O y z$ plane and is tilted by the angle $\varphi$ with respect to the axis $O y$.

It is assumed that the medium is homogeneous and isotropic. Waves are generated by a source with a predetermined frequency $\omega$. Thus, the wave amplitude can only be reduced if the wave number is the complex value: $k=\eta-i \xi$.

The Landau-Lifshitz equation with the dissipative term of the Gilbert form is used to describe the motion of the magnetization vector $\vec{m}$ in a lossy medium (with the damping coefficient $\alpha$ ). In this geometry the dispersion equation for MSSW has the form:

$$
\tanh (k d \vartheta)=\frac{2 \mu \vartheta}{\beta}
$$

In (1) we have one constant parameter $d$, four variables: $k, \mu$ is the magnetic permeability of the medium of the form:

$$
\mu=1+\frac{\Omega_{H}+i \alpha \Omega}{\Omega_{H}^{2}-\left(1+\alpha^{2}\right) \Omega^{2}+i 2 \alpha \Omega \Omega_{H}},
$$

and two ancillary quantities $\vartheta$ and $\beta$, which are expressed by formulae

$$
\vartheta=\sqrt{\cos ^{2} \varphi-\frac{\sin ^{2} \varphi}{\mu}}, \quad \beta=\left(\nu^{2}-\mu^{2}+\mu\right) \cos ^{2} \varphi-\mu,
$$

where $\nu$ is the magnetic susceptibility of the medium having the form:

$$
\nu=\frac{\Omega}{\Omega_{H}^{2}-\left(1+\alpha^{2}\right) \Omega^{2}+i 2 \alpha \Omega \Omega_{H}} .
$$

The quantities $\Omega$ and $\Omega_{H}$ given in equations (2) and (4) are the normalized wave frequency and the magnetic field respectively:

$$
\Omega=\frac{\omega}{4 \pi \gamma M}, \quad \Omega_{H}=\frac{H}{4 \pi M},
$$

where $\gamma$ is the gyromagnetic ratio.

\section{Analysis}

Fig. 1 demonstrates the assemblage of dispersion curves for the real and imaginary parts of the wave number obtained by the numerical solution of equation (1). To plot Fig. 1 the following parameters were used: $4 \pi M=1750 \mathrm{Gs}, H=437.5 \mathrm{Oe}, d=15 \mathrm{mkm}, \gamma=1.76 \cdot 10^{7} \mathrm{rad} /(\mathrm{s} \cdot \mathrm{Gs})$. A set of solid curves represents the dependence of the wave frequency from the real part of the wave number $f(\eta)$; the family of dashed curves displays the dependence of the frequency from the imaginary part of the wave number $f(\xi)$. Each line in the set is constructed for different values of the damping parameter $\alpha$.

As Fig. 1 indicates, either set of curves is separated into two subset branches corresponding to dispersion of forward (MSFSW) and backward (MSBSW) waves. Between the MSFSW and MSBSW branches of the dispersion curves $f(\eta)$ there is a bandgap of width $\Delta f$ that is the increasing function of the damping coefficient. Moreover, the frequency range of MSFSW and 


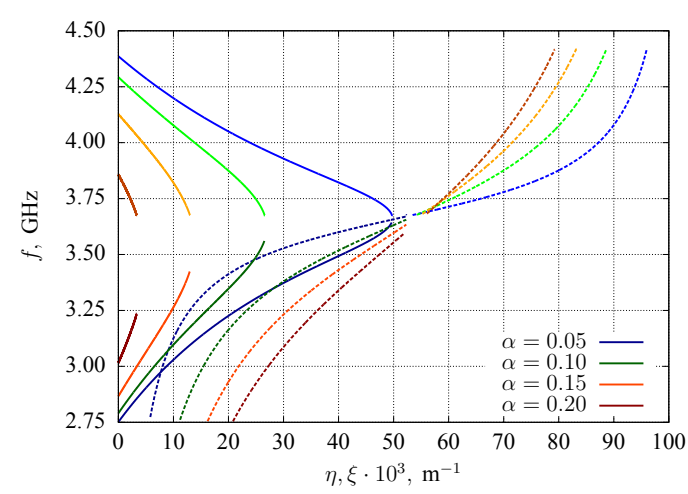

(a) $\varphi=0^{\circ}$

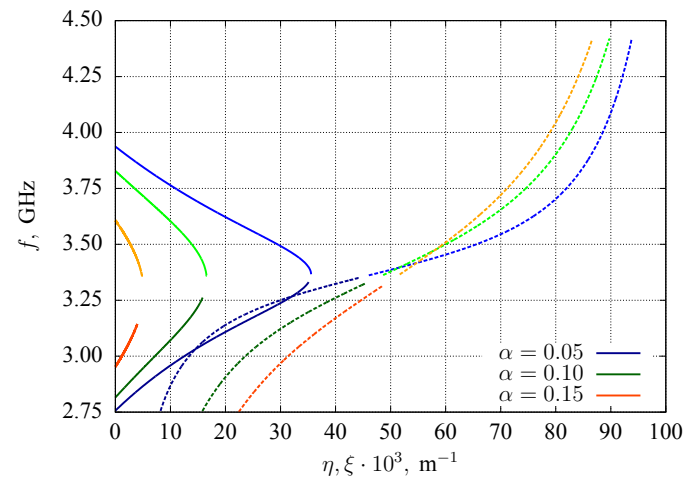

(c) $\varphi=30^{\circ}$

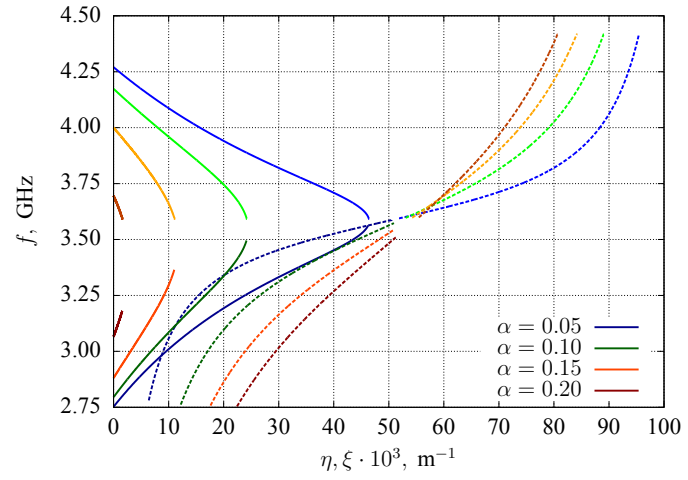

(b) $\varphi=15^{\circ}$

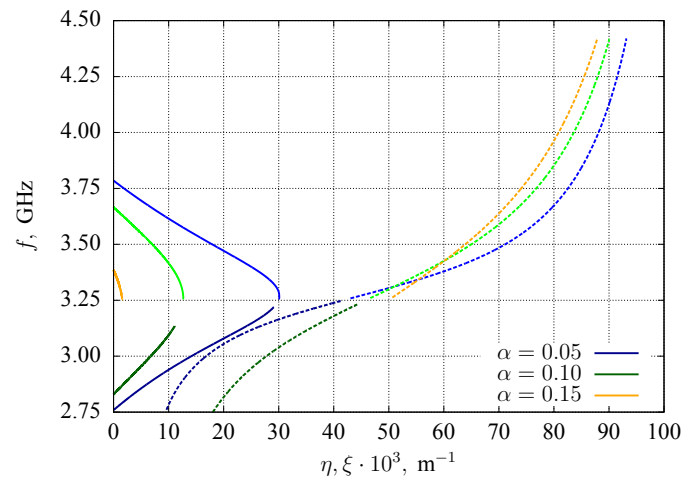

(d) $\varphi=35^{\circ}$

Fig. 1. Dispersion curves of the MSFSW and MSBSW with different values of $\alpha$ and $\varphi$. Solid curves $-f(\eta)$; dashed $-f(\xi)$.

MSBSW significantly narrows with the increase of $\alpha$. Besides, there is a limitation to the maximum of $\eta$ for a given $\alpha$.

The dispersion curves for the dissipation $f(\xi)$ show that $\xi$ is a monotonically increasing function of the wave frequency, and with an increase of $\alpha$ this dependence becomes more linear.

MSSW dispersion characteristics depend essentially on the direction of the wave propagation. Four special cases corresponding to different values of angles $\varphi$ are represented in Fig. 1. Fig. 1(a) illustrates the case when the wave propagates directly perpendicularly to magnetic field direction. In this case all characteristics of MSFSW and MSBSW dispersion mentiond above are clearly occur. Fig. 1(b) displays the case of wave propagation in the direction of straight tilted by the angle $\varphi=15^{\circ}$ with respect to the axis $O y$. The basic regularities in this case are the same as for case $\varphi=0^{\circ}$, however the restrictions of wave number and frequency are more enhanced. For example, when $\alpha=0.20$, this results in decreasing of the frequency spectrum and the maximum wave number of MSFSW and MSBSW on about a half. This features are not so clear under smaller values of the damping parameter, e.g. if $\alpha=0.05$, the frequency spectrum and the maximum wave number of MSFSW and MSBSW are reduced for about $10 \%$. Fig. 1(c) demonstrates, that with the growth of $\varphi$ for $15^{\circ}$ the frequency spectrum and the maximum wave number of MSFSW and MSBSW keep decreasing. This leads to fact, that MSFSW and MSBSW can not exist under $\alpha=0.20$. Fig. 1(d) shows that in further increasing of $\varphi$ for $5^{\circ}$ MSFSW can not propagate any more in film with $\alpha=0.15$, while MSBSW still can be represented. 


\section{Conclusions}

In this research dispersion relations for the real and imaginary parts of the wave number were obtained. It was shown that dispersion curves are limited both by the wave number and frequency, and these restrictions tighten with an increase in the damping parameter.

MSFSW and MSBSW are characterised by existence of a critical value of the damping parameter and propagation angle, beyond which it does not exist. With the increase of the angle $\varphi$, limitation of dispersion curves tightens, the branches of the dispersion curves are shifted towards lower wave numbers and the damping parameter limits them from below and above.

\section{References}

[1] W.S.Ishak, Magnetostatic wave technology: a review, Proceedings of the IEEE, 76(1988), no. $2,171-187$.

[2] Y.Kajiwara et al., Transmission of electrical signals by spin-wave interconversion in a magnetic insulator, Nature, 464(2010), 262-266.

[3] O.Kolokoltsev et al., Hot spin-wave resonators and scatterers, J. of Appl. Phys., 112(2012), 013902 .

[4] S.Jun, et al., Parametric and modulation instabilities of magnetostatic surface spin waves in ferromagnetic films, J. of Appl. Phys., 81(1997), 1341.

[5] R.W.Damon, J.R.Eshbach, Magnetostatic modes of a ferromagnet slab, J. Phys. Chem. Solids, 19(1961), no. 3-4, 308-320.

[6] K.L.Wong et al., Unidirectional propagation of magnetostatic surface spin waves at a magnetic film surface, Appl. Phys. Lett., 105(2014), 232403.

[7] J.H.Kwon et al., Characterization of magnetostatic surface spin waves in magnetic thin films: evaluation for microelectronic applications, Appl. Phys. A, 111(2013), 369-378.

\section{Влияние диссипации на дисперсию поверхностных магнитостатических волн}

Павел А. Макаров

Институт точных наук и информационных технологий Сыктывкарский государственный университет Октябрьский пр., 55, Сыктывкар, 167001

Россия

Владимир И. Щеглов

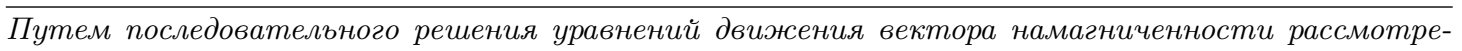
но влияние диссипации на поверхностные магнитостатические волны, распространяющиеся 8 плоскости продольно намагниченной ферритовой пластины. Получены дисперсионные соотношения для действительной и мнимой частей волнового числа. Показано, что дисперсионные кривые ограничены как по волновому числу, так и по частоте, и эти ограничения усиливаются с увеличением параметра затухания. И для прямых, и для обратных волн имеются критические значения параметра затухания и угла распространения, начиная с которых они не существуют.

Ключевые слова: дисперсия, ферриты, магнитостатические волны, диссипация. 